\title{
Solución numérica del flujo transitorio en tanques cilíndricos horizontales producido por la inyección de aire con tres toberas*
}

\section{Arturo Lizardi-Ramos \\ Hilario Terres-Peña \\ Raymundo López-Callejas \\ Mabel Vaca-Mier \\ Sandra Chávez-Sánchez}

\section{Universidad Autónoma Metropolitana \\ Unidad Azcapotzalco \\ Departamento de Energía}

Av. San Pablo No. 180, Col. Reynosa Tamaulipas, CP 02200,

Alcaldía Azcapotzalco, Ciudad de México.

MÉXICO

$$
\begin{array}{r}
\text { correo electrónico (email): } \\
\text { arlr@azc.uam.mx } \\
\text { tph@azc.uam.mx } \\
\text { rlc@azc.uam.mx } \\
\text { mvm_Icar@hotmail.com } \\
\text { scs@azc.uam.mx }
\end{array}
$$

Recibido 11-02-2020, aceptado 30-04-2020.

\section{Resumen}

Se presenta el análisis numérico transitorio del flujo generado por la inyección de aire en un cilindro horizontal que contiene agua y que su parte superior está abierta a la atmósfera. El aire es introducido al depósito por medio de tres toberas y frente a ellas se colocan dos tipos de deflector; con sección transversal circular y triangular. El modelo matemático contempla las ecuaciones de Reynolds-Navier-Stokes en coordenadas cilíndricas para un fluido newtoniano, viscoso, en dos fases, en régimen turbulento y en estado transitorio; y se resuelve con el método numérico del elemento finito. Los resultados muestran los campos de velocidad transitorios en la sección transversal del recipiente que coincide con el centro de las toberas que inyectan el aire al contenedor. También se analiza el comportamiento de la velocidad azimutal a lo largo del diámetro del cilindro para diferentes tiempos y para

*Este artículo es la versión extendida de los mejores trabajos del Congreso Nacional de Ingeniería Electromecánica y de Sistemas 2019 la posición axial antes mencionada. Al comparar el campo de velocidades en estado permanente del sistema con deflectores triangulares contra el circular se encontró que: a) el valor del vector velocidad en la parte superior izquierda del tanque disminuyó $3.20 \%$; b) el valor máximo positivo promedio de la velocidad azimutal a lo largo del diámetro del depósito disminuyó 3.71\%.

Palabras clave: deflectores, flujo radial, velocidad azimutal.

\section{Abstract \\ (Numerical Solution of the Transient Flow in Horizon- tal Cylindrical Tanks Produced by the Injection of Air with Three Nozzles)}

The numerical transient analysis of the flow generated by the injection of air in a horizontal cylinder that contains water and is open to the atmosphere, is presented. The air is introduced with three nozzles and, in front of them, two types of baffles with circular and triangular cross-section are placed. The mathematical model considers the Reynolds-Navier-Stokes equations in cylindrical coordinates for a two-phase viscous Newtonian fluid, in the turbulent regime, and the transitory state; and it is solved using the numerical finite element method. The results show the transient velocity fields in the crosssection of the container which coincides with the center of the nozzles that inject the air into the container. The behavior of azimuthal velocity along the diameter of the cylinder for different times and the aforementioned axial position is also analyzed. By comparing the fields of velocity in a steady state of the system with triangular baffles against circular ones it was found that: (a) the value of the velocity vector in the left upper part of the tank diminished $3.20 \%$; and (b) the maximum average positive value of the azimuth velocity along the diameter of the container diminished $3.71 \%$.

Index terms: deflectors, radial flow, azimuth velocity.

\section{Nomenclatura}

$C$, calor específico $(\mathrm{J} / \mathrm{kg} \mathrm{K})$

$C_{d}$, coeficiente de arrastre

$d_{b}$, diámetro de la burbuja $(\mathrm{m})$

F, vector fuerza de cuerpo $\left(\mathrm{N} / \mathrm{m}^{3}\right)$ 
$g$, aceleración gravitatoria $\left(\mathrm{m} / \mathrm{s}^{2}\right)$

$k$, conductividad térmica $(\mathrm{W} / \mathrm{m} \mathrm{K})$

$m_{g l}$, tasa de transferencia de masa $\left(\mathrm{kg} / \mathrm{s} \mathrm{m}^{3}\right)$

$M_{w}$, peso molecular de la burbuja de gas $(\mathrm{kg} / \mathrm{kmol})$

$-\mathbf{n}$, vector unitario normal

$\mathbf{N}_{\mathrm{g}}$, vector flujo de masa de gas $\left(\mathrm{kg} / \mathrm{m}^{2} \mathrm{~s}\right)$

$P$, presión $(\mathrm{Pa})$

$R u$, constante universal de los gases $(\mathrm{J} / \mathrm{mol} \mathrm{K})$

$r$, coordenada radial $(\mathrm{m})$

Re, número de Reynolds

$t$, tiempo (s)

$T$, temperatura $(\mathrm{K})$

$u$, componente de velocidad $\mathrm{radial}(\mathrm{m} / \mathrm{s})$

$v$, componente de velocidad tangencial $(\mathrm{m} / \mathrm{s})$

$\mathbf{V}_{\mathrm{g}}$, velocidad del gas $(\mathrm{m} / \mathrm{s})$

$\mathbf{V}_{1}$, velocidad de la fase líquida $(\mathrm{m} / \mathrm{s})$

$\mathbf{V}_{\text {des }}$, velocidad de deslizamiento $(\mathrm{m} / \mathrm{s})$

$\mathbf{V}_{\text {in }}$, velocidad del gas a la entrada $(\mathrm{m} / \mathrm{s})$

$w$, componente de velocidad axial $(\mathrm{m} / \mathrm{s})$

$z$, coordenada axial $(\mathrm{m})$

$\beta$, coeficiente de expansión volumétrico $(\mathrm{K}-1)$

$\rho_{\mathrm{l}}$, densidad del líquido $\left(\mathrm{kg} / \mathrm{m}^{3}\right)$

$\rho_{\mathrm{g}}$, densidad del gas $\left(\mathrm{kg} / \mathrm{m}^{3}\right)$

$\rho_{\text {in }}$, densidad del gas a la entrada $\left(\mathrm{kg} / \mathrm{m}^{3}\right)$

$\mu_{1}$, viscosidad dinámica de la fase líquida $(\mathrm{kg} / \mathrm{m} \mathrm{s})$

$\mu_{\mathrm{T}}$, viscosidad turbulenta $(\mathrm{kg} / \mathrm{ms})$

$\theta$, coordenada tangencial (grados)

$\Theta_{1}$, fracción de volumen líquido $\left(\mathrm{m}^{3} / \mathrm{m}^{3}\right)$

$\Theta_{\mathrm{g}}$, fracción de volumen de gas $\left(\mathrm{m}^{3} / \mathrm{m}^{3}\right)$

\section{Introducción}

El movimiento de líquidos en el interior de recipientes cilíndricos horizontales, generado por la inyección de aire a través de sus fronteras, es un fenómeno que interesa por el gran número de aplicaciones industriales. Por ejemplo, en acuicultura se persigue la eliminación de substancias inertes, la destrucción de gérmenes patógenos y además facilitar el intercambio de oxígeno del aire a la fase líquida del agua. Por lo anterior, para la acuicultura es prudente mantener el agua de los tanques tan próxima como sea posible a la saturación total de oxígeno disuelto $(100 \%)$ y para ello se requiere un proceso de aireación. Existen varios métodos para este fin, uno de ellos consiste en inyectar el aire que viene de un compresor a través de una tubería que tiene en un extremo una tobera, al interior del depósito [1]. En el sector agrícola, por ejemplo, se utilizan contenedores cilíndricos que almacenan fertilizantes y que se instalan en los cabezales de riego donde se realizan las diferentes disoluciones de abonos químicos para su aplicación junto con el riego. El número de tanques que hay en una instalación depende del uso que requiere la explotación agrícola, siendo lo más usual la instalación de cuatro depósitos para fertilizantes y uno para aportaciones especiales como ácidos o componentes orgánicos. A su vez, los contenedores llevan sistemas de agitación para mantener disueltos los fertilizantes. Estos pueden ser de dos tipos: a) agitadores mecánicos: compuestos por un motor cuyo eje provoca la rotación de una hélice que agita la disolución del tanque, b) agitadores neumáticos: compuestos por una bomba soplante conectada a un sistema de tuberías instalada en los depósitos que inyecta aire en la disolución, provocando la agitación de la misma, World Fertilizer use Manual [2]. Otro proceso de inyección de aire en contenedores cilíndricos, que ha sido estudiado ampliamente, se encuentra en los convertidores de cobre donde se requiere de la inyección de aire para oxidar las impurezas del mismo. Por ejemplo, A. Valencia et al. [3] estudiaron numéricamente la dinámica de fluidos dentro de un recipiente con agua (con la geometría de un convertidor de cobre) debido a la inyección de aire provocada por una tobera sumergida. La simulación tridimensional, dependiente del tiempo, se llevó a cabo con el software Fluent de CFD. Se compararon los resultados numéricos y experimentales de la velocidad de entrada del aire en el depósito que produce los resultados más favorables respecto a una buena mezcla en el recipiente con un mínimo de salpicaduras. C. Real et al. [4] caracterizaron un convertidor de cobre con inyección de aire en el fondo, utilizando el software Fluent. En el planteamiento del problema contemplaron las ecuaciones de NavierStokes en flujo transitorio, para múltiples velocidades de inyección de aire, y en condiciones isotérmicas. En dicho trabajo se encontró que a medida que se aumentaba la velocidad del aire inyectado, se incrementaba la energía cinética de la mata de cobre de manera casi lineal. D. K. Chibwe et al. [5], analizaron experimental y numéricamente el patrón de flujo y mezclado en un convertidor industrial Peirce-Smith, empleando un modelo en frío. Se llevaron a cabo simulaciones 2-D y 3-D del sistema de tres fases. La capa de mata y escoria fueron simuladas con agua y queroseno respectivamente. Los resultados revelaron que tanto la tasa de flujo volumétrico del aire como la presencia de la capa de escoria superpuesta tienen efectos profundos sobre la eficiencia de mezcla del convertidor. Otra aplicación, pero para el caso de flujo con gases es la de T. Fernández, M. Toledo, J. F. Vázquez [6], ellos analizaron el movimiento de un torbellino generado por un ventilador axial de velocidad variable dentro de un tubo que tiene una relación longitud/diámetro $(x / d)$ de 7 . Se determinó que la intensidad del torbellino disminuye con el incremento del número de Reynolds y que la correlación más adecuada entre las variables es del tipo lineal. Asimismo, se encontró que existe una analogía entre el incremento de la distancia axial con el incremento del número de Reynolds.

En este trabajo se pretenden obtener los campos de velocidad transitorios generados por la inyección de aire en un cilindro horizontal que contiene agua y cuya superficie supe- 
rior está abierta a la atmósfera. El aire es inyectado por tres toberas y frente a ellas se colocan dos tipos de deflector: con sección transversal circular y triangular. Para ello se resuelven las ecuaciones de Reynolds-Navier-Stokes en coordenadas polares cilíndricas, en estado transitorio, junto con las condiciones iniciales y de frontera adecuadas. Los resultados permitirán obtener los campos de velocidad transitorios en la sección transversal del recipiente que coincide con el centro de las toberas que inyectan el aire al contenedor, así como el comportamiento de la velocidad azimutal $(v)$ a lo largo del diámetro del cilindro para diferentes tiempos.

\section{Problema por analizar}

El sistema a analizar consta de un depósito cilíndrico horizontal de radio $\mathrm{R} 1=100 \mathrm{~mm}$ y longitud $\mathrm{L} 1=100 \mathrm{~mm}$, cuyo interior tiene agua. El recipiente cuenta con una abertura en la parte superior de L2 $=28.2 \mathrm{~mm}$ de ancho que permite la interacción con la atmósfera. El aire se introduce radialmente al tanque a través de tres toberas de radio $\mathrm{R} 2=2 \mathrm{~mm}$ colocadas en la parte media de la longitud del cilindro y a un ángulo de $\theta 1=225^{\circ}$, $\theta 2=270^{\circ}$ y $\theta 3=315^{\circ}$, respectivamente, Fig. 1 y 2 . Para mejorar la distribución de aire en el tanque se colocan deflectores enfrente de cada tobera. Los dos tipos de deflector empleados son de sección transversal: circular de radio $\mathrm{R} 3=5 \mathrm{~mm}$ y triangular de lado L3 = $8.66 \mathrm{~mm}$, Fig. 3. Los deflectores se colocan enfrente a las toberas a una distancia de R4 $=97 \mathrm{~mm}$ desde el centro del depósito.

\section{Planteamiento matemático}

La formulación matemática se basa en las ecuaciones de Reynolds-Navier-Stokes (RANS) que se pueden emplear para determinar el flujo de un fluido Newtoniano, viscoso, bifásico,

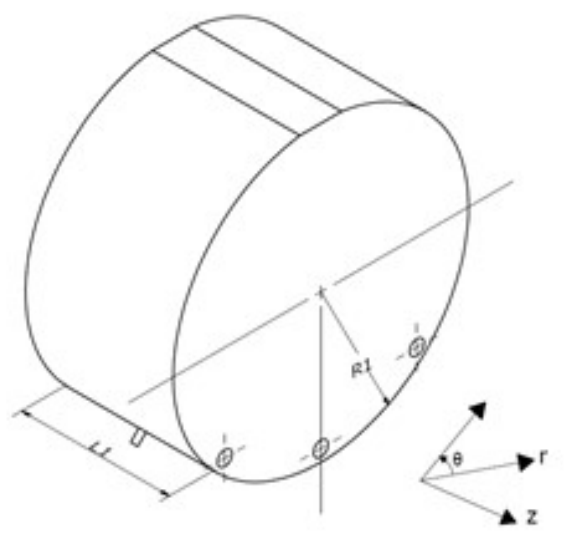

Fig. 1. Vista 3D del sistema en estudio.

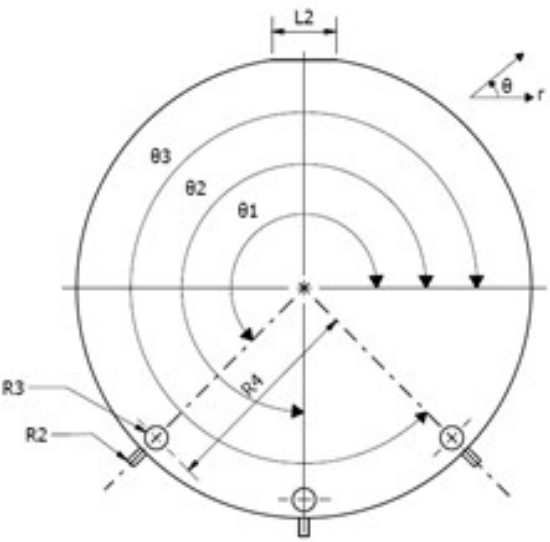

Fig. 2. Vista frontal del sistema en estudio.

en régimen turbulento y en estado transitorio, en el interior de un recipiente cilíndrico, [7], [8], [9]. Para la fase líquida:

$\varphi_{1} \rho_{1} \frac{\partial \vec{V}_{1}}{\partial t}+\varphi_{1} \rho_{I} \vec{V}_{1} \cdot \nabla \vec{V}_{1}=-\nabla P+\nabla \cdot\left[\varphi_{1}\left(\mu_{1}+\mu_{T}\right)\left(\nabla \vec{V}_{1}+\left(\nabla \vec{V}_{1}\right)^{T}\right)\right]+\varphi_{1} \rho_{1} g+\vec{F}$

Sabiendo que para bajas concentraciones de gas, la fracción de volumen líquido $\phi_{1}$ es aproximadamente uno, entonces:

$$
\nabla \cdot \vec{V}_{1}=0
$$

La ecuación para la fase gaseosa es:

$$
\frac{\partial \rho_{g} \varphi_{g}}{\partial t}+\nabla \cdot\left(\rho_{g} \varphi_{g} \vec{V}_{g}\right)=-m_{g l}
$$

Pero se considera que no hay transferencia de masa entre la fase líquida y gaseosa, por lo que $m_{g l}=0$. Así, la ecuación (3) queda:

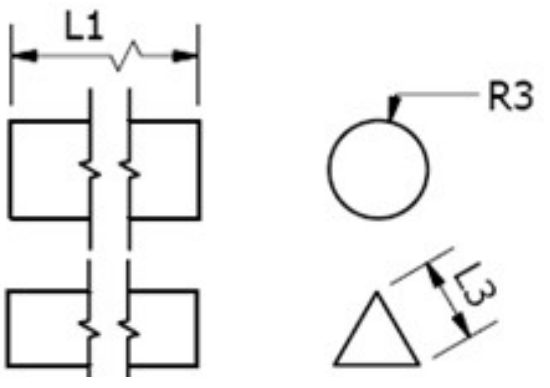

Fig. 3. Tipos de deflector empleados. 


$$
\frac{\partial \rho_{\mathrm{g}} \varphi_{\mathrm{g}}}{\partial t}+\nabla \cdot\left(\rho_{\mathrm{g}} \varphi_{\mathrm{g}} \vec{V}_{\mathrm{g}}\right)=0
$$

La densidad del gas se calcula a partir de la ecuación de estado del gas ideal:

$$
\rho_{\mathrm{g}}=\frac{P M_{w}}{R u T}
$$

Aquí $M_{w}$ es el peso molecular de la burbuja de gas, $R u=8.314$ $\mathrm{J} / \mathrm{mol}, K$ es la constante universal de los gases y $T=293 \mathrm{~K}$ la temperatura del gas. La velocidad del gas se puede calcular a partir de la ecuación $\mathbf{V}_{\mathrm{g}}=\mathbf{V}_{1}+\mathbf{V}_{\text {des }}$, donde $\mathbf{V}_{\text {des }}$ es la velocidad de deslizamiento entre el agua y el aire. El balance presiónarrastre se emplea para calcular $\mathbf{V}_{\text {des }}$. El modelo se basa en la suposición de que las fuerzas de presión de las burbujas se equilibran con la fuerza de arrastre:

$$
\frac{3 C_{d}}{4 d_{b}} \rho_{\mathrm{I}}\left|\overrightarrow{\mathrm{V}}_{\mathrm{des}}\right| \overrightarrow{\mathrm{V}}_{\mathrm{des}}=-\nabla P
$$

Donde $d_{b}=0.001 \mathrm{~m}$ es el diámetro de la burbuja y $C_{d}$ es el coeficiente de arrastre (adimensional). Debido a que el diámetro de burbuja es inferior a $0.002 \mathrm{~m}$, se usó el modelo de coeficiente de arrastre de Hadamard-Rybczynski [7], [10].

$$
C_{d}=\frac{16}{R e}
$$

El número de Reynolds se calcula con:

$$
R e=\frac{d_{b} \rho_{1} / \overrightarrow{\mathrm{V}}_{\text {des }} /}{\mu_{\mathrm{l}}}
$$

Para las condiciones de frontera se tomó la condición de no deslizamiento, $\mathbf{V}_{1}=0$, en las paredes internas del depósito y sobre los deflectores. Para la entrada del aire se considera que $-\mathbf{n} \mathbf{N}_{\mathrm{g}}=\mathbf{V}_{\text {in }} \rho_{\text {in }}$ donde $-\mathbf{n}$ es un vector normal a la sección de entrada que "apunta" al interior del cilindro. El valor de la velocidad del aire a la entrada se tomó como $\mathbf{V}_{\text {in }}=0.1 \mathrm{~m} / \mathrm{s}$ y la densidad como $\rho_{\text {in }}=0.973 \mathrm{~kg} / \mathrm{m}^{3}$. Para la salida del aire se consideró la condición de no deslizamiento, $\mathbf{n V}_{1}=0$, la cual supone la condición de no penetración del agua pero si del aire. Finalmente, las ecuaciones (1) y (4) se resuelven para obtener las variables $\mathbf{V}_{1}$ y $P$. Del campo de velocidades $\mathbf{V}_{1}$ se obtienen las tres componentes escalares: radial, $u$, tangencial, $v, \mathrm{y}$ axial, $w$.

\section{Planteamiento numérico}

Para resolver las ecuaciones que definen el movimiento del fluido en el interior del recipiente se empleó el método numérico del elemento finito. Para ello se utilizó el software COMSOL Multiphysics, el cual es un programa de simulación multi-plataforma que permite hacer estudios complejos basados en la física del problema y que son acoplados a sistemas de ecuaciones diferenciales parciales. La metodología usada fue [11], [12]:

a) Se selecciona en el software el modelo 3D.

b) Se dibuja la geometría a analizar. Para ello se generó un cilindro horizontal de $100 \mathrm{~mm}$ de radio por $100 \mathrm{~mm}$ de longitud, con una abertura en la parte superior de $28.2 \mathrm{~mm}$ de ancho a todo lo largo del depósito. Para introducir el aire al tanque, que se encuentra lleno de agua, se colocaron tres toberas de $2 \mathrm{~mm}$ de radio, ubicadas a la mitad de la longitud del cilindro y a un ángulo de $225^{\circ}, 270^{\circ}$ y $315^{\circ}$, respecto a la horizontal. Se generaron dos sistemas con deflectores de diferente sección transversal: uno circular de $5 \mathrm{~mm}$ de radio y uno triangular de $8.66 \mathrm{~mm}$ de lado. Los deflectores se colocaron enfrente a las toberas a una distancia de $97 \mathrm{~mm}$ medido desde el centro del recipiente.

c) Se analiza la independencia del tamaño de la malla y su relación con los resultados. Para ambos sistemas se generaron diferentes tipos de malla con distintos números de elementos. En el arreglo con deflectores de sección transversal circular y triangular, las mallas que dieron los mejores resultados fueron las de 135,840 y 213,000 elementos, para el primer caso, y de 92,191 y 210,000 elementos, para el segundo caso. La diferencia entre los resultados de ambos mallados fue de tan solo $0.19 \%$ y de $0.25 \%$, respectivamente. Así que, considerando los recursos de cómputo disponibles y el tiempo de convergencia de la solución, se eligieron las mallas con 135,840 y 92,191 elementos, Fig. 4 y 5.

d) Se introduce el valor de las propiedades del agua y aire: viscosidad dinámica $(\mu)$, densidad $(\rho)$, calor específico $(C)$, coeficiente de expansión volumétrico $(\beta)$ y conductividad térmica $(k)$. La presión en el sistema se modela con el algoritmo de relajación (TDMA).

e) Se introducen las condiciones de frontera y se da el valor de la velocidad de entrada del aire, en este caso $\mathbf{V}_{\text {in }}=0.1 \mathrm{~m} / \mathrm{s}$. La interfaz es resuelta con el código de flujo burbujeante, turbulento y bifásico, que es el adecuado para resolver las ecuaciones descritas en este trabajo.

f) Se configura la solución de paso segregada con un error de $1.0 \times 10^{-8}$ como criterio de convergencia.

g) Se corre el programa y se obtienen los valores transitorios de los campos del vector velocidad $\left(\mathbf{V}_{1}\right)$ y de la componente de velocidad azimutal $(v)$. 


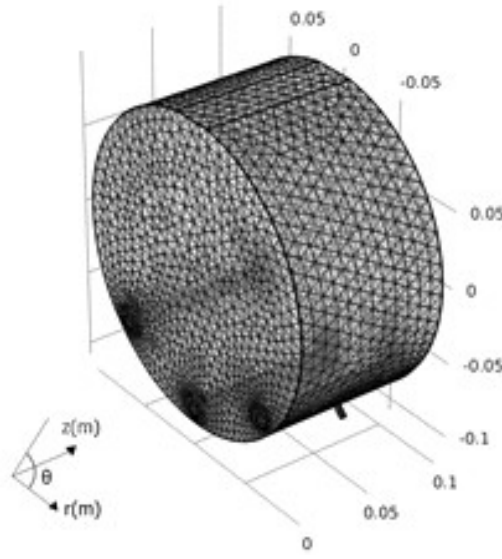

Fig. 4. Malla del tanque con deflectores de sección transversal circular.

\section{Análisis y discusión de resultados}

Una vez hecha la programación, se llevaron a cabo las corridas para los dos arreglos planteados. El programa se dejó correr con un paso temporal de 0.5 segundos desde $0 \mathrm{~s}$ hasta un tiempo de $10 \mathrm{~min}$, observándose que a los $60 \mathrm{~s}$ se había alcanzado el estado permanente. Lo anterior se concluyó al comparar la diferencia entre los resultados de los tiempos $40 \mathrm{~s}$ y $50 \mathrm{~s}$, y entre 50 s y 60 s, siendo esta de 0.71 y $0.67 \%$, respectivamente. De la Fig. 6 a la 11 se muestran los campos del vector velocidad para la los dos casos a analizar y para los tiempos de $1 \mathrm{~s}$, $10 \mathrm{~s}$ y $60 \mathrm{~s}$. En dichas figuras se resalta el valor del vector velocidad en cuatro posiciones de interés, ubicados de acuerdo con el sistema de referencia de la Fig. 2 , ( $\left.\mathrm{R} \mathrm{mm}, \theta^{\circ}\right)$, en: (45.5, $\left.90^{\circ}\right),\left(91,120^{\circ}\right),\left(91,180^{\circ}\right)$ y $\left(45.5,270^{\circ}\right)$, lo anterior con el propósito de comparar su magnitud al cambiar el tiempo y el tipo de

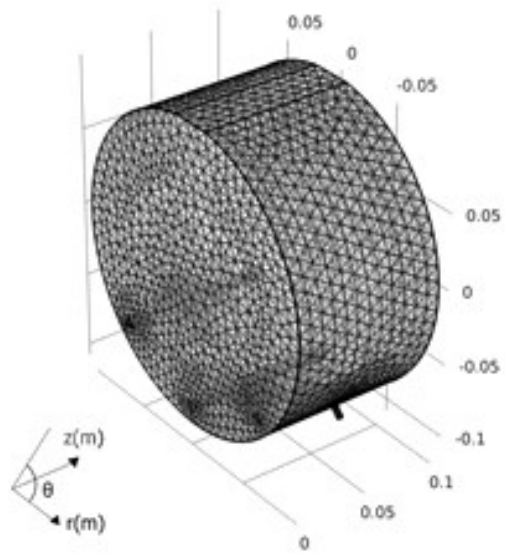

Fig. 5. Malla del tanque con deflectores de sección transversal triangular.

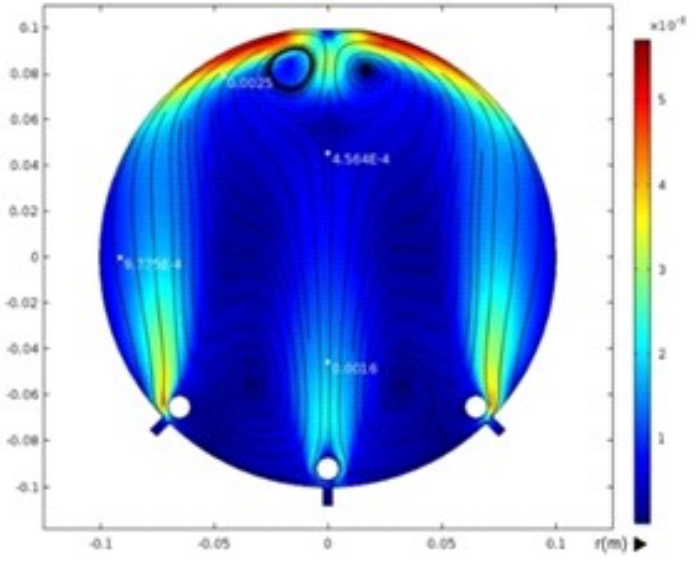

Fig. 6. Campo de velocidades con deflectores circulares, $t=1 \mathrm{~s}$.

deflector. Es importante señalar que se está analizando el movimiento del fluido dentro del contenedor sin tomar en cuenta la concentración aire-agua que se tiene en el sistema, es decir, que el flujo de aire solo se toma en cuenta como el causante del movimiento.

En la Fig. 6 se muestra el arreglo con deflectores circulares y tiempo $1 \mathrm{~s}$. Se observa que el aire se introduce al recipiente, se incorpora al agua y rodea a los deflectores. El flujo se mueve principalmente hacia la parte externa de los deflectores laterales y hacia el centro en el deflector inferior. En la parte superior del cilindro se tienen los valores más altos de la velocidad $\left(2.5 \times 10^{-3} \mathrm{~m} / \mathrm{s}\right)$ y en la zona lateral se presentan bajas magnitudes $\left(0.98 \times 10^{-3} \mathrm{~m} / \mathrm{s}\right)$. En la Fig. 7 , que muestra el tiempo de $10 \mathrm{~s}$, se aprecia que el flujo inducido a través de las toberas ha comenzado a formar dos vórtices secundarios en

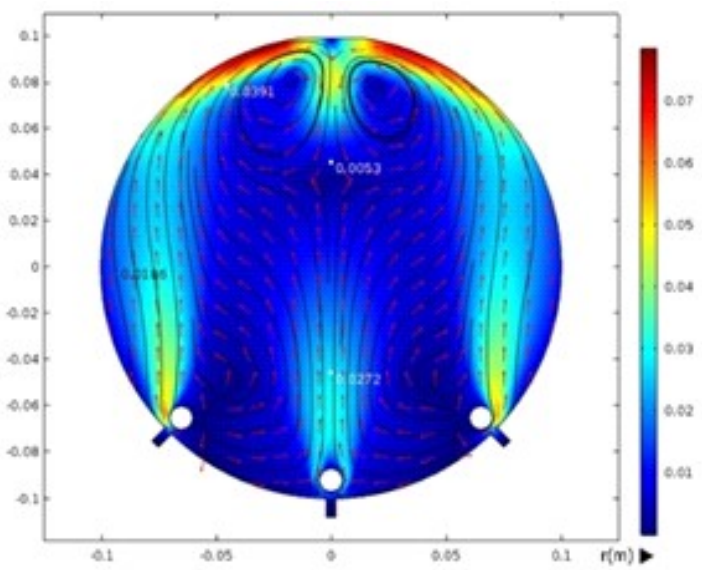

Fig. 7. Campo de velocidades con deflectores circulares, $t=10 \mathrm{~s}$. 


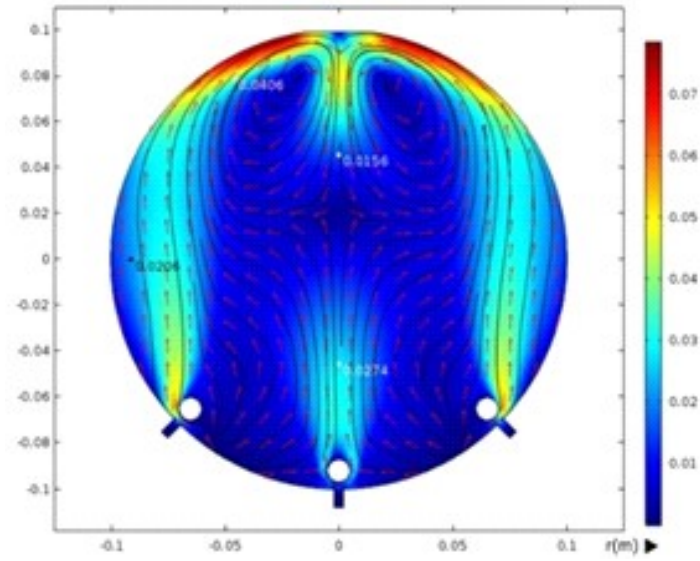

Fig. 8. Campo de velocidades con deflectores circulares, $t=60 \mathrm{~s}$.

la parte superior del recipiente. El de lado izquierdo gira en sentido horario y el lado derecho gira en sentido anti horario. Nuevamente, como en el caso anterior, se tienen los valores más altos de la velocidad en la parte superior del depósito $\left(39.1 \times 10^{-3}\right)$ y disminuyen sus magnitudes en la parte lateral $\left(18.6 \times 10^{-3} \mathrm{~m} / \mathrm{s}\right)$. Finalmente, en la Fig. 8 se muestra el tiempo de $60 \mathrm{~s}$, es decir, cuando se ha alcanzado el estado permanente. Se aprecia que los dos flujos rotatorios ya están bien definidos. La velocidad en la parte superior del recipiente toma el valor de $40.6 \times 10^{-3}$ y en la parte lateral de $20.6 \times 10^{-3} \mathrm{~m} / \mathrm{s}$.

Por otro lado, en las Fig. 9, 10 y 11, se muestra el arreglo con deflectores triangulares para un tiempo de 1,10 y $60 \mathrm{~s}$. Se aprecia que el comportamiento es parecido al caso anterior, pero con magnitudes distintas. En la Tabla 1 se muestran, a manera de resumen, los valores de la velocidad para las ubi-

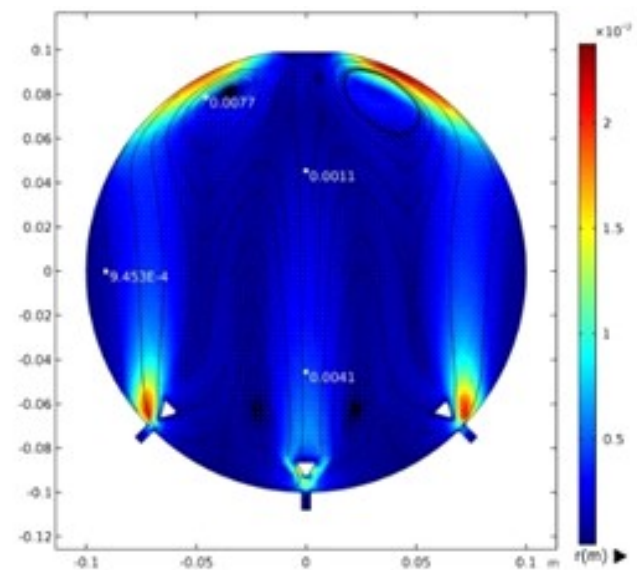

Fig. 9. Campo de velocidades con deflectores triangulares, $t=1 \mathrm{~s}$.

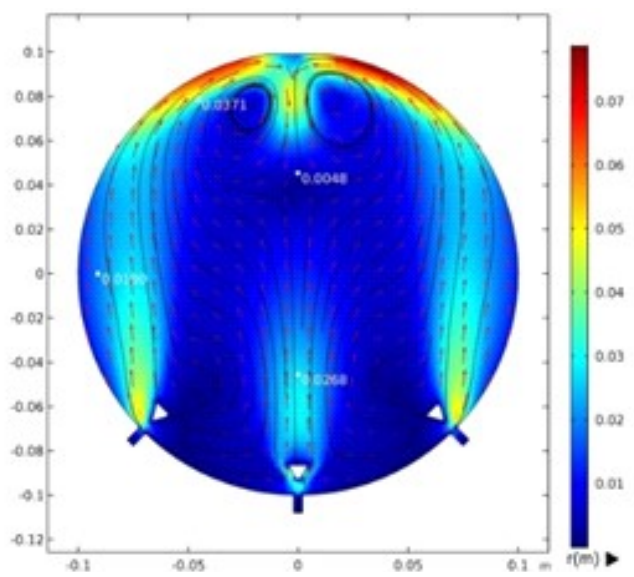

Fig. 10. Campo de velocidades con deflectores triangulares, $t=10 \mathrm{~s}$.

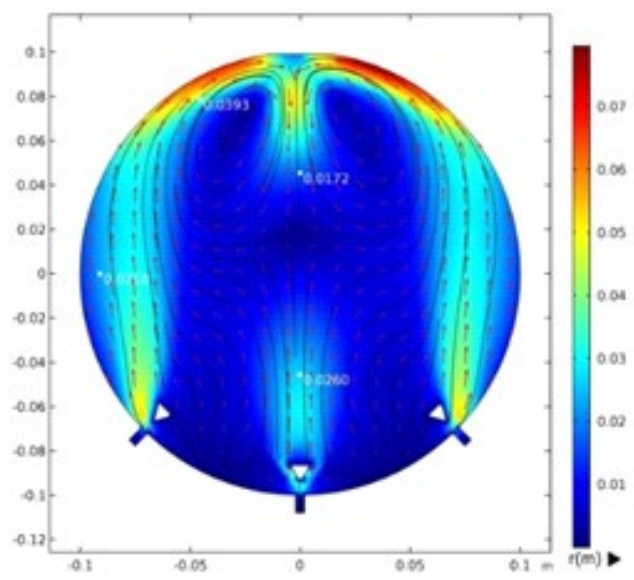

Fig. 11. Campo de velocidades con deflectores triangulares, $t=60 \mathrm{~s}$.

caciones $\left(91 \mathrm{~mm}, 120^{\circ}\right)$ y $\left(91 \mathrm{~mm}, 180^{\circ}\right)$, para los dos arreglos y para los tres tiempos.

Comparando la magnitud del vector velocidad en la parte superior del tanque, para el tiempo de $60 \mathrm{~s}$, del sistema con deflectores triangulares respecto a los circulares, se observó una disminución del 3.20\%. Es decir que en esta parte del depósito se tiene una mayor velocidad cuando se colocan los deflectores circulares a comparación de los triangulares.

Tabla 1. Vector velocidad $\left(\mathbf{V}_{1}, \mathrm{~m} / \mathrm{s}\right)$ en los dos arreglos y para las posiciones seleccionadas.

\begin{tabular}{cll} 
Tiempo (s) & Deflector circular & Deflector triangular \\
1 & $2.5 \times 10^{-3}, 0.98 \times 10^{-3}$ & $7.7 \times 10^{-3}, 0.94 \times 10^{-3}$ \\
10 & $39.1 \times 10^{-3}, 18.6 \times 10^{-3}$ & $37.1 \times 10^{-3}, 19.0 \times 10^{-3}$ \\
60 & $40.6 \times 10^{-3}, 20.6 \times 10^{-3}$ & $39.3 \times 10^{-3}, 21.8 \times 10^{-3}$ \\
\hline
\end{tabular}




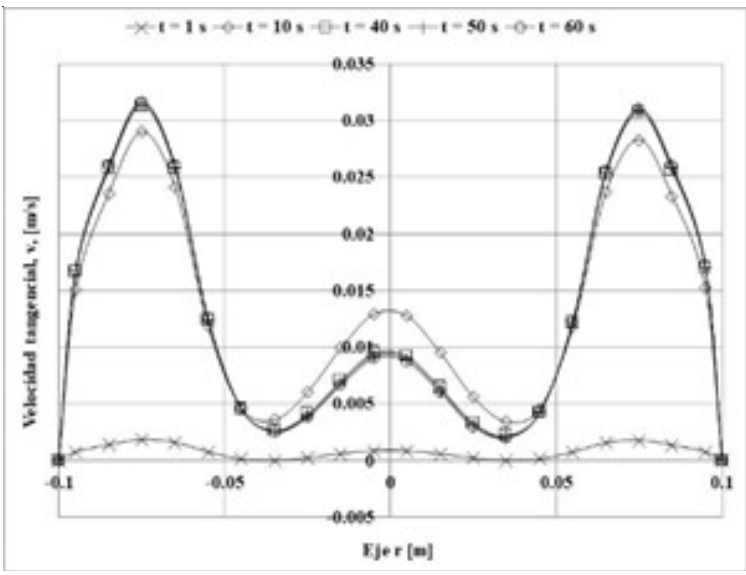

Fig. 12. Velocidad tangencial ( $v$ ) para arreglo con deflectores circulares.

Para analizar más detalladamente el movimiento del fluido en los dos arreglos, se elaboraron gráficas de la componente de velocidad tangencial $(v)$ a lo largo de la línea horizontal que va de los $0^{\circ}$ hasta $180^{\circ}$, y para los tiempos de $1,10,40,50$ y 60 segundos. En la Fig. 12 se presenta la distribución de velocidades del sistema con deflectores circulares para los cinco tiempos. Para el tiempo de $1 \mathrm{~s}$, se aprecia que la curva de la componente de velocidad tangencial comienza en cero sobre la pared izquierda del cilindro, posteriormente va aumentando su valor hasta un máximo positivo de $1.81 \times 10^{-3} \mathrm{~m} / \mathrm{s}$, de allí baja su intensidad, pero se vuelve a presentar un incremento de su magnitud hasta otro máximo positivo de $0.84 \times 10^{-3}$. Después de este punto la velocidad nuevamente vuelve a decrecer y a aumentar su valor hasta un máximo positivo de $1.75 \times 10^{-3} \mathrm{~m} / \mathrm{s}$. Finalmente disminuye su magnitud hasta hacerse cero sobre la pared derecha del tanque. Este comportamiento es correcto debido a la

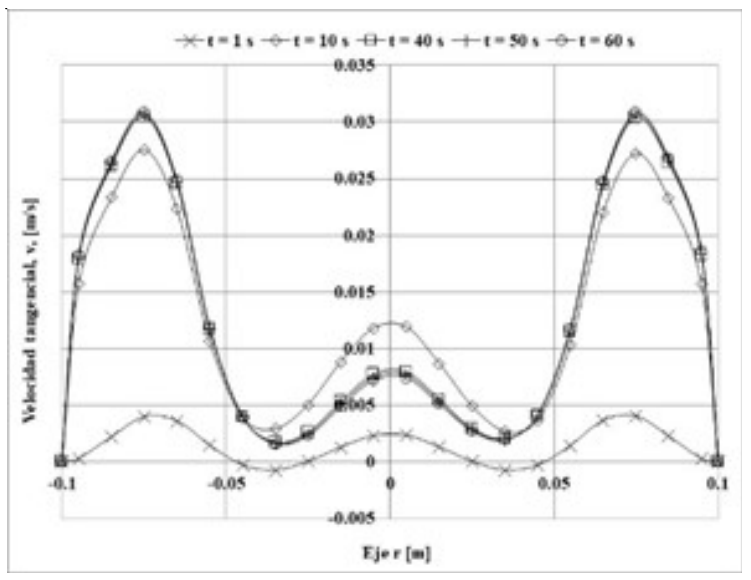

Fig. 13. Velocidad tangencial (v) para arreglo con deflectores triangulares.
Tabla 2. Valores máximos positivos de la velocidad tangencial $(\mathrm{v}, \mathrm{m} / \mathrm{s})$ para los dos arreglos.

$\begin{array}{ccc}\text { Tiempo (s) } & \text { Circular } & \text { Triangular } \\ 1 & 1.81 \times 10^{-3}, 0.84 \times 10^{-3}, & 3.97 \times 10^{-3}, 2.33 \times 10^{-3}, \\ & 1.75 \times 10^{-3} & 4.04 \times 10^{-3} \\ 10 & 28.97 \times 10^{-3}, 12.92 \times 10^{-3}, & 27.54 \times 10^{-3}, 11.89 \times 10^{-3}, \\ & 28.30 \times 10^{-3} & 27.21 \times 10^{-3} \\ 60 & 31.62 \times 10^{-3}, 9.00 \times 10^{-3}, & 30.82 \times 10^{-3}, 7.37 \times 10^{-3} \\ & 31.03 \times 10^{-3} & 30.79 \times 10^{-3} \\ \end{array}$

simetría que presenta el modelo físico. En el tiempo de $10 \mathrm{~s}$, tal como se mostró anteriormente en el campo de velocidades, es cuando se empiezan a formar dos flujos circulatorios en la parte superior del arreglo. Estos flujos provocan en la velocidad tangencial la formación de tres máximos positivos con valores de: $28.97 \times 10^{-3}, 12.92 \times 10^{-3}$ y $28.30 \times 10^{-3} \mathrm{~m} / \mathrm{s}$. Las tres toberas inducen el movimiento del fluido hacia la parte superior del depósito, ocasionando la formación de estas magnitudes máximas. Finalmente, para el tiempo de $60 \mathrm{~s}$, los flujos circulatorios han alcanzado el estado permanente. Para este tiempo la velocidad tangencial también tiene tres valores máximos positivos, estos son de: $31.62 \times 10^{-3}, 9.00 \times 10^{-3}$ y $31.03 \times 10^{-3} \mathrm{~m} / \mathrm{s}$. Cabe mencionar que solo se han colocado las curvas de 40, $50 \mathrm{~s}$ para mostrar que el tiempo de $60 \mathrm{~s}$ corresponde al estado permanente; esto se comprueba al observar que en estos tiempos las curvas prácticamente se empalman.

En la Fig. 13 se presenta la distribución de velocidades para el sistema con deflectores triangulares y para los tiempos de 1, 10, 40, 50 y 60 segundos. En ella se observa un comportamiento parecido al caso anterior, pero con magnitudes distintas. En la Tabla 2 se indican los valores máximos de la velocidad tangencial para los dos arreglos estudiados y para tres tiempos.

Comparando las magnitudes máximas positivas promedio de la componente de velocidad tangencial del arreglo con deflectores triangulares respecto a los circulares, se encontró una disminución del 3.71\%. Es decir, el flujo con mayor intensidad se tiene con los deflectores circulares. Estos resultados tienen concordancia con el análisis de los campos de velocidad antes descritos.

\section{Conclusiones}

En este trabajo se ha analizado el campo de velocidades y el comportamiento de la componente de velocidad tangencial, dependientes del tiempo, que se producen por la inyección de aire, a través de tres toberas, en un depósito cilíndrico 
horizontal que contiene agua, que está abierto a la atmósfera, y al cual se le colocaron deflectores de sección transversal: circular y triangular, enfrente de las toberas. Tanto el método numérico como la metodología propuesta para la solución fueron adecuados, pues permitieron obtener los campos de velocidad transitorios de los arreglos planteados, así como el comportamiento de la velocidad tangencial a lo largo del diámetro del recipiente, encontrándose concordancia con resultados de la literatura. Al comparar el valor del vector velocidad en la parte superior del tanque, para el tiempo de $60 \mathrm{~s}$, del sistema con deflectores triangulares respecto a los circulares, se observó una disminución del 3.20\%. Por otro lado, al hacer la misma comparación pero de las magnitudes máximas positivas de la velocidad tangencial promedio, se encontró una reducción del 3.71\%. Ambos resultados mostraron que los deflectores circulares son los que tienen mejor desempeño en la distribución del movimiento del fluido en el interior del tanque, aunque la diferencia que hubo entre ambos fue pequeña. Aunque se obtuvieron buenos resultados, aún falta mayor investigación para considerar otras variables de interés, tales como otros rangos de velocidad, distintos tipos de fluido, etc.

\section{Referencias}

[1] O. Galli-Merino, F. Miguel-Sal, Sistemas de recirculación y tratamiento de agua, Secretaría de Agricultura, Ganadería, Pesca y Alimentos CENADAC, Santa AnaCorrientes, Argentina, 2007. [en linea]. Disponible en: http://www.minagri.gob.ar/sitio/areas/acuicultura/ cultivos/otros/. Consultado: 10 enero 2019.

[2] D. Halliday, M. Trenkel, IFA world fertilizer use manual, París: IFA, 1992. [en línea]. Disponible en: https:// www.fertilizer.org/. Consultado: 25 marzo 2019.

[3] A. Valencia, R. Paredes, M. Rosales, E. Godoy, J. Ortega, "Fluid dynamics of submerged gas injection into liquid in a model of copper converter," International Communications in Heat and Mass Transfer, vol. 31, núm. 1, pp. 21-30, enero, 2004. [en línea]. Disponible en: https://doi.org/10.1016/S0735-1933(03)00198-2. Consultado: 18 febrero 2019.

[4] C. Real, L. Hoyos, F. Cervantes, R. Miranda., M. PalomarPardave, M. Barron, J. Gonzales. "Fluid characterization of copper converters," Asociación Argentina de Mecánica Computacional, vol. 26, núm. 15, pp. 13111323, octubre, 2007. [en línea]. Disponible en: https:// cimec.org.ar/ojs/index.php/mc/article/view/1124/1075. Consultado: 03 marzo, 2019.

[5] D. Chibwe, G. Akdogan, C. Aldrich, R. Eric, "CFD modelling of global mixing parameters in a Peirce-Smith converter with comparison to physical modelling," Chemical Product and Process Modeling, vol. 6, núm. 1, pp. 1-27, enero, 2011. [en línea]. Disponible en: https:// espace.curtin.edu.au/bitstream/handle/20.500.11937/ 39656/204637.pdf?sequence=2. Consultado: 19 abril 2019.

[6] T. Fernández, M. Toledo, J. F. Vázquez, "Variación de la intensidad del torbellino con el número de Reynolds", Científica, vol.14, núm. 4, pp. 173-178, octubre-dic. 2010.

[7] M. AL-Mashhadani, S. Wilkinson, W. Zimmerman, "Airlift bioreactor for biological applications with microbubble mediated transport processes," Chemical Engineering Science, vol. 137, pp. 243-253, diciembre, 2015. [en línea]. Disponible en: https://doi.org/10.1016/ j.ces.2015.06.032. Consultado:25 abril 2019.

[8] A. N. Tijonov, A. A. Samarsky, Ecuaciones de la física matemática, Moscú: MIR, 1980. [en línea]. Disponible en: http://samarskii.ru/books/book1984.pdf. Consultado: 19 marzo 2019.

[9] L. D. Landau, E. M. Lisfshitz, Fluids Mechanics, $2^{\text {a }}$ ed., Cambridge: Pergamon Press, 1987. [en línea]. Disponible en: https://www.elsevier.com/books/fluid-mechanics/ landau/978-0-08-033933-7. Consultado: 08 mayo 2019.

[10] C. Crowe, M. Sommerfeld and Y. Tsuji, Multiphase Flows with Droplets and Particles, $2^{\mathrm{a}}$ ed, Boca Raton, Florida: CRC Press, 1998. [en linea]. Disponible en: https:// www.crcpress.com/Multiphase-Flows-with-Dropletsand-Particles/Crowe-Schwarzkopf-Sommerfeld-Tsuji/p/ book/9781439840504. Consultado: 26 abril 2019

[11] C. R. Torres, B. J. Grau. Introducción a la mecánica de fluidos y transferencia de calor con COMSOL Multiphysics, $1^{\mathrm{a}}$ ed., Barcelona: Addlink Media, 2007. [en línea]. Disponible en: https://www.addlink.es/ productos/introduccion-a-la-mecanica-de-fluidos-ytransferencia-de-calor-con-comsol-multiphysics. Consultado: 25 enero 2019.

[12] R. W. Pryor, Multiphysics modeling using COMSOL: a first principles approach, Boston: Jones \& Bartlett Learning, 2011. [en línea]. Diponible en: http:// teguhhady.lecturer.pens.ac.id/Multiphysics\% 20modeling\%20using\%20COMSOL.pdf. Consultado: 28 enero 2019. 
